\title{
Cecal bascule: Report of a
}

case

\author{
LARRY JAY FEINMAN, D.O. \\ STANLEY D. KOLMAN, D.O \\ ANTHONY A. MINISSALE, D.O., FACOS \\ VIVIAN M. BARSKY, D.O., FACOS \\ Philadelphia, Pennsylvania \\ ELLIOT B. FRANK, D.O. \\ Huntingdon Valley, Pennsylvania
}

\begin{abstract}
Cecal bascule is a rare type
of cecal volvulus in which the cecum

folds anteriorly on the ascending colon

such that its posterior surface comes

anterior and the appendix is displaced

to the right upper quadrant. Cecal

volvulus presents with colon

obstruction, and the diagnosis can be

readily made with barium enema and

abdominal plain films. However,

although patients with cecal bascule

may present with colon obstruction, the

diagnosis is not easily made

preoperatively and is usually only made

at laparotomy. Once diagnosed, a

number of treatments are available, both

operative and nonoperative. We report

on a patient with a cecal bascule and

outline the presentation and

management of this anomaly.
\end{abstract}

Volvulus of the cecum can occur in three ways: (1) with rotation obliquely, with the cecum lying in the left upper abdomen; (2) with rotation about the long axis of the cecum; and (3) as the bascule type. ${ }^{1}$ In the cecal bascule, the cecum folds anteriorly on the ascending colon so that the posterior surface becomes anterior and the appendix becomes uppermost. ${ }^{2,3}$ If the ileocecal valve is competent, the bascule becomes a closed-loop obstruction. ${ }^{3}$

For a cecal bascule to form, certain factors must be present. There must be an absence or elongation of the mesentery and a point of fixation to act as a fulcrum. ${ }^{4}$ The point of fixation can be normal ascending colon with attached mesentery, an adhesive band, or even a chronically inflamed appendix, as described by Weinstein. ${ }^{1}$ In addition to congenital abnormalities that predispose to bascule formation, certain contributory factors usually are present, most commonly previous abdominal surgery ${ }^{5}$ or pregnancy. ${ }^{6}$ Barium enema can cause volvulus in patients so predisposed. ${ }^{7}$
In the case reported here, the patient presented with symptoms of bowel obstruction and x-ray and colonoscopic findings suggestive of cecal torsion. At laparotomy, a cecal bascule was found and right hemicolectomy was performed.

\section{Report of case}

A 49-year-old white woman was admitted through the emergency room with a 4-day history of periumbilical pain and 36 hours of nausea and vomiting. She denied hematemesis or melena, recent viral illness, alcohol consumption, and prior medical or surgical history. The systems review was negative.

With the exception of the abdominal examination, physical examination was unremarkable. The patient's upper abdomen was distended and tympanitic. Bowel sounds were present and normal. Her right upper quadrant was tender, but no guarding or rebound tenderness was noted. Digital rectal examination was normal; however, the stools tested heme-positive.

Laboratory results of note included a leukocyte count of $13,800 / \mathrm{cu}$. mm., with a differential count of 86 percent polymorphonuclear leucocytes, 1 percent band forms, and 13 percent lymphocytes. The hemoglobin concentration was $16.0 \mathrm{gm} . / \mathrm{dl}$., and the hematocrit value was 48.2 percent. Serum electrolyte, blood urea nitrogen, and serum creatinine levels and urinalysis results were normal. The electrocardiogram was unremarkable.

The chest $\mathrm{x}$-ray was normal, while the abdominal films showed a markedly distended stomach (Fig. 1). Ultrasonographic examination of the abdomen demonstrated a large, solid mass in the left upper abdomen. Barium enema revealed a mechanical obstruction secondary to an intraluminal filling defect at the proximal transverse colon (Fig. 2).

Colonoscopy was performed and demonstrated marked angulation at the proximal transverse colon. This corresponded to the barium enema finding of a filling defect, thereby raising the possibility of torsion.

At laparotomy, a cecal bascule was found. The cecum was flipped up onto the ascending colon into the right upper quadrant, so that the posterior surface was anterior and the appendix was uppermost. The cecum was completely obstructed at the fold, and, due to an almost completely patent ileocecal valve, the cecum measured 

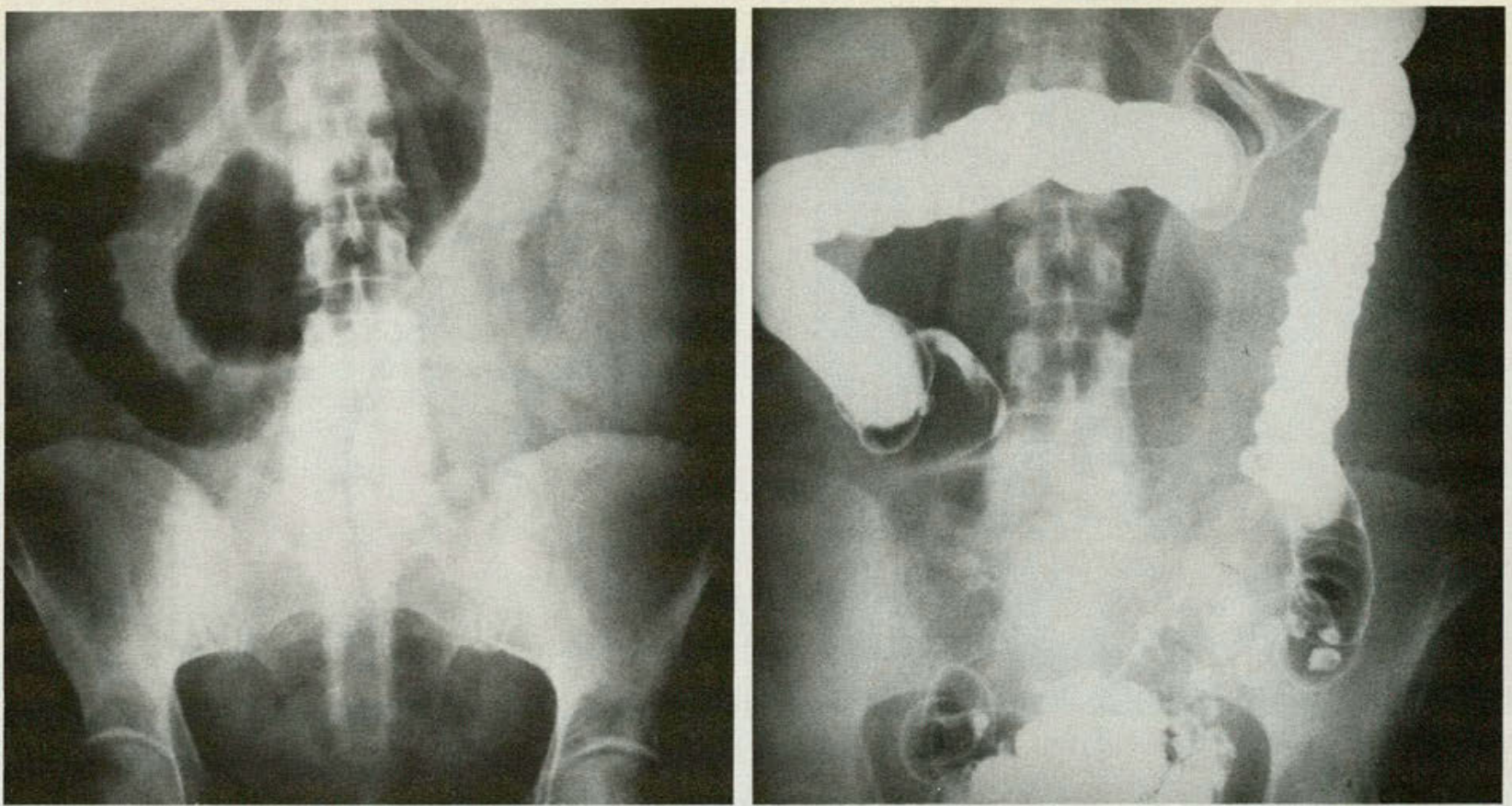

Fig. 1 Abdominal plain film shows a markedly distended stomach. Fig. 2 Barium enema reveals a mechanical obstruction secondary to an intramural filling defect at the proximal transverse colon.

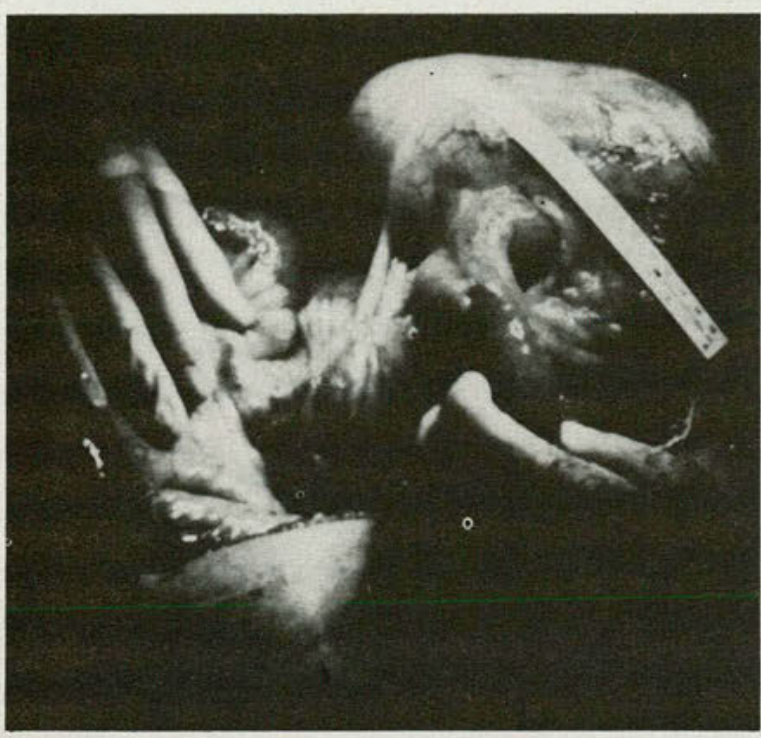

Fig. 3 The cecum, measuring $25 \mathrm{~cm}$. in diameter, is completely obstructed at the fold. Fig. 3A The illustration at right shows the obstructed cecum.

$25 \mathrm{~cm}$. across (Fig. 3). The cecum was noted to have no peritoneal attachment, and the ascending colon was attached with a very sparse, lateral, quite abnormal reflection. The mesentery was twisted and bruised (Fig. 4). The patient underwent right hemicolectomy with ileotransverse anastomosis and had an uneventful recovery. She was discharged from the hospital on the tenth postoperative day.

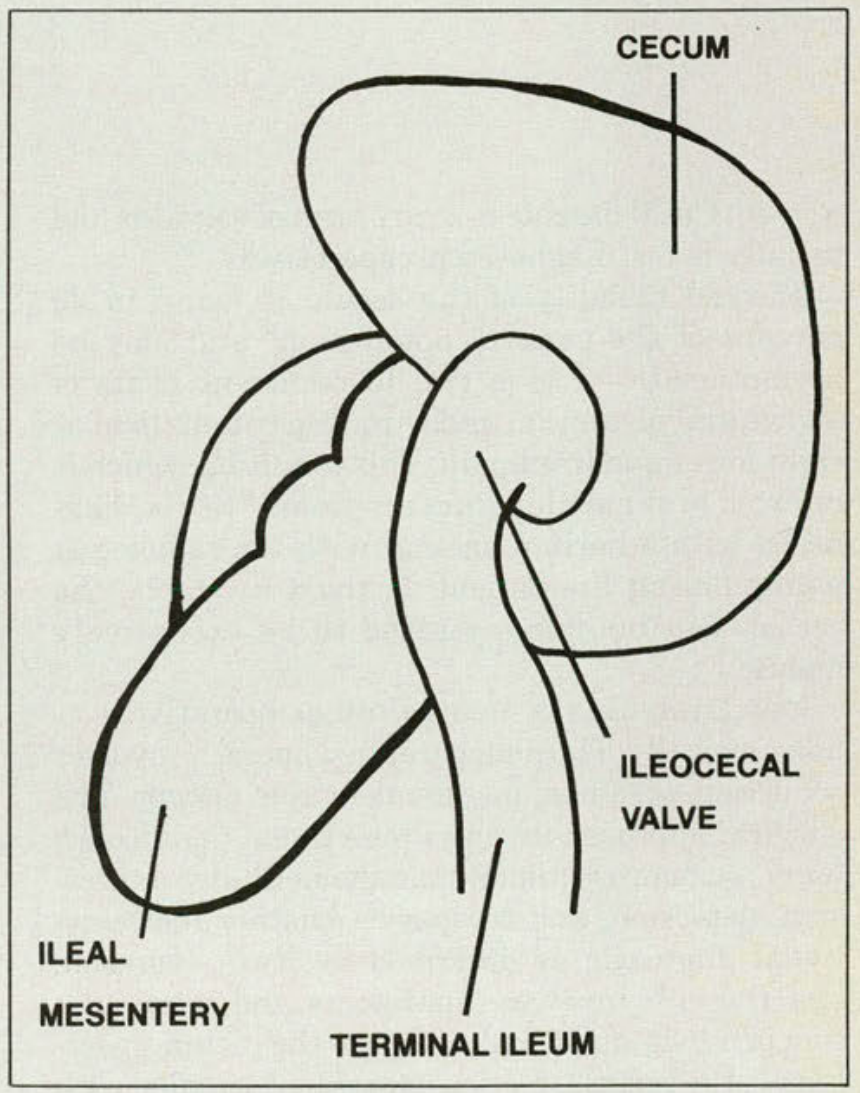

Discussion

Cecal volvulus must be suspected in patients who present with abdominal pain and distention. Without a high index of suspicion, volvulus can easily be 


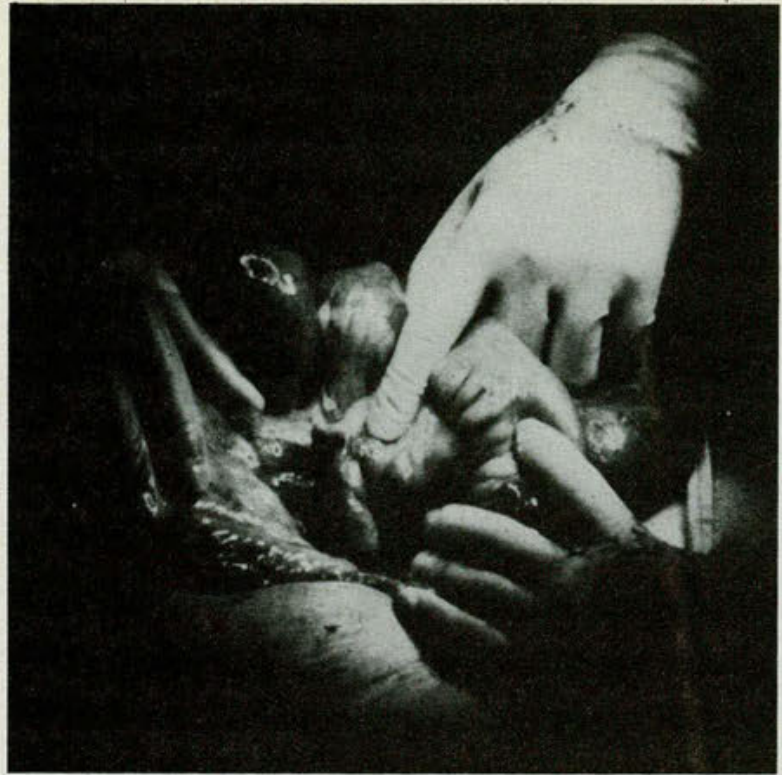

Fig. 4 In this view of the cecal mesentery, it appears bruised and twisted. Fig. 4A Drawing at right depicts the extent of the twisted mesentery.

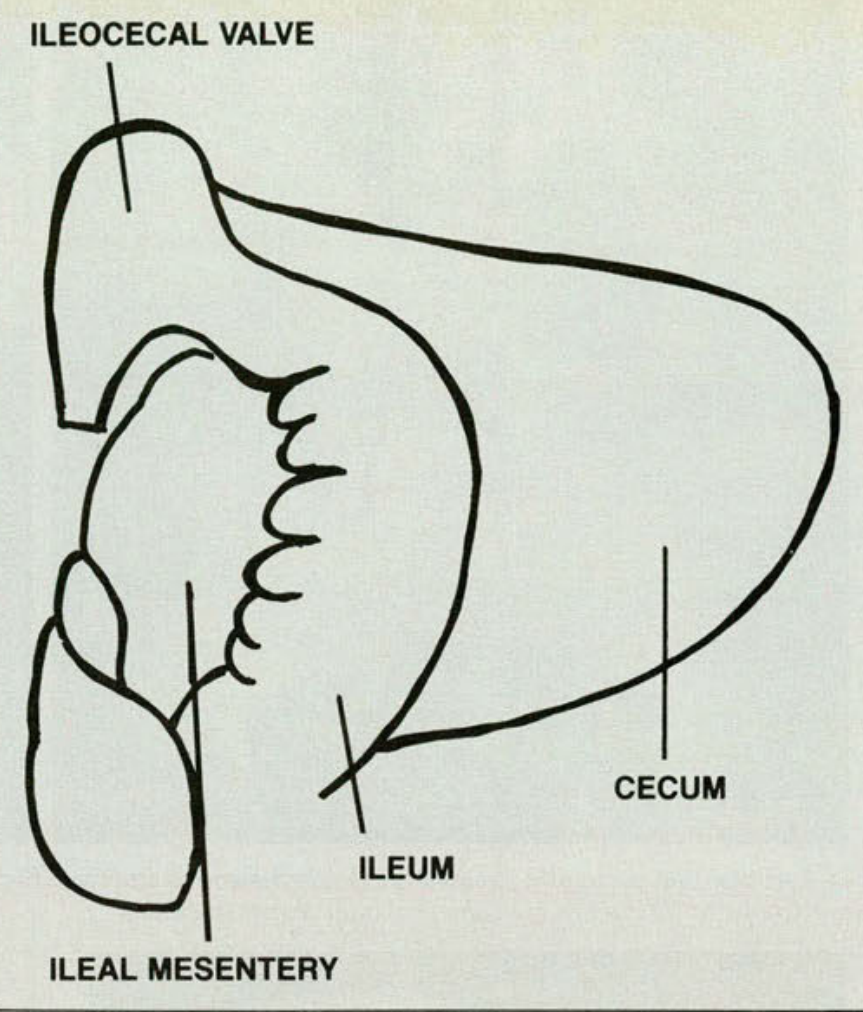

missed. Cecal bascule is a rare form of volvulus and usually is not diagnosed preoperatively.

Marked mobility of the cecum is found in 20 percent of the general population ${ }^{8}$ and may be asymptomatic or give rise to recurrent bouts of abdominal distention and chronic periumbilical or right lower quadrant pain. This condition, which is referred to as mobile cecum syndrome,${ }^{9}$ can be diagnosed with a barium enema. With the radiologist manipulating the patient on the x-ray table, the cecum can be demonstrated to be excessively mobile. ${ }^{9}$

Cecal volvulus is treated either operatively or nonsurgically. The nonoperative approach involves reduction with barium enema or colonoscopy. The surgical approach involves resectional (hemicolectomy) or nonresectional management (decompression, detorsion, and cecopexy ${ }^{5}$ ).Another nonresectional approach, as described by Ryan, Johnson, and Baker, ${ }^{10}$ involves laparotomy and manipulation of a long intestinal tube into the cecum (inserted via the rectum) for decompression, coupled with cecopexy.

\footnotetext{
1. Weinstein, M.: Volvulus of the cecum and ascending colon. Ann Surg 107:248-59, Feb 38

2. Bobroff, L.M., et al.: The cecal bascule. Am J Roentgenol 115:249-52.
} Jun 72
3. Rabin, M.S., and Richter, I.A.: Caecal bascule. A potential clinical and radiological pitfall. Case reports. S Afr Med J 54:242-4, 5 Aug 78

4. Govoni, A.F.: The caecal bascule revisited. Radiol Diagn 25:143-53, 1984

5. Weiss, B.D.: Cecal volvulus. A diagnostic and therapeutic challenge. Postgrad Med 72:189-94, Aug 82

6. Pratt, A.T., et al.: Cecal volvulus in pregnancy. Obstet Gynecol 57S:37-40, Jun 81

7. Hemingway, A.P.: Caecal volvulus. A new twist to the barium enema Br J Radiol 53:806-7, Aug 80

8. Chalfant, S.A.: Torsion of the cecum with review of the literature and report of a case. Am J Obstet Gynecol 2:597-600, Dec 21

9. Rogers, R.L., and Harford, F.J.: Mobile cecum syndrome. Dis Colon Rectum 27:399-402, Jun 84

10. Ryan, J.A., Jr., Johnson, M.G., and Baker, J.W.: Operative treatment of cecal volvulus combining cecopexy with intestinal tube decompression. Surg Gynecol Obstet 160:84-6, Jan 85

Accepted for publication in April 1986. Updating, as necessary, has been done by the authors.

At the time this paper was written, Dr. Feinman was a chief resident in general surgery at Metropolitan Hospital, Parkview Division. He is now a fellow in vascular surgery at Metropolitan Hospital. Drs. Kolman and Barsky are in private practice of general surgery at Metropolitan Hospital, Parkview Division. Dr. Minissale is chairman of the Department of Surgery and residency trainer; and Dr. Frank is in the private practice of gastroenterology.

Dr. Feinman, 8947 Fairfield Street, Philadelphia, Pennsylvania 19152. 